\title{
Mining Multi Drug-Pathways via A Probabilistic Heterogeneous Network Multi-label Classifier
}

\author{
Taysir Hassan A. Soliman
}

\begin{abstract}
Mining drug networks is a very important research issue to discover hidden relations between multi drug-entities relations, such as multi drug-pathways, multi drug-targets, and multi drug-diseases. One very important relation is the drug-pathway, where drugs affect the human body through their pathways. In this paper, a probabilistic Heterogeneous Network Multi-label Classifier (HNMC) is proposed to classify multi drug-pathways relations. Data is collected from Drugbank.ca [1], Kegg (keg drug, Kegg diseases, Kegg pathways, Kegg orthologs, Kegg brite) [2] and small molecular pathways [3,4]. For drug-pathways data, two datasets are considered: one is based on Drug-Drug Interaction (DDI) and the other is based on Drug-Pathways Interactions (DPI). HNMC has proved its efficiency with an average of $90 \%$ precision, $92.35 \%$ recall, $92 \%$ accuracy, and $96 \%$ ROC.
\end{abstract}

Keywords--- Multi Drug-Pathways Prediction, Probabilistic Heterogeneous Networks and Multi-label Classification

\section{INTRODUCTION}

$\mathrm{T}$ HERE is a critical need to better model and understand how the complex interactions between drugs and their targets as well as their pathways contribute to drug efficacy and possible side effects [5]. Complex interactions can be modeled as drug networks contain very crucial information, including their targets, enzymes, carriers, transporters, pathways, diseases, and organisms, as illustrated in Figure 1. To understand the relations between such entities will help to do the following:

1. Derive better disease treatment and 2) avoid side effects. Takigawa et al [6] summarizes the importance of polypharmacology or drug promiscuity of drug networks because of the following reasons: 1) Exquisitely selective drugs for a single target are likely to exhibit low clinical efficacy and be unsuccessful [7].

2. Multi-targeted drugs have been clinically successful, particularly as dual or multiplex kinase inhibitors [8]. 3) Many approved drugs, potentially the majority of

Taysir Hassan A. Soliman, Associate Professor, Information Systems, Faculty of Computers \& Information, Assiut University, Egypt. E-mail: taysser.soliman@fci.au.edu.eg

DOI: 10.9756/BIJRCE.10368 therapeutic agents, are less selective than initially thought [9]. 4) The robustness of biological systems can be implied by the scale-free nature of drug-target networks [10,11].

Large datasets are available in both the literature, such as PubChem [12] and PubMed [13] and in public databases, such as Drugbank.ca [1], Drugbank.com [14], SIDER [15], CHEBI [16], PharmGKB [17], and KEGG [2] (KEGG DISEASE, KEGG DRUG, KEGG LIGAND, KEGG PATHWAY, and KEGG BRITE). It is a complex process in terms of both handling available large datasets and developing computational methods for predicting new evolving drug different entity-relations. A recent concentration has been made on drug-target prediction because it can help to reduce the time, cost, and failure rates for developing new drugs. Recently, with the accumulation of drug-related data sets related to drug side effects and pharmacological data, it has become possible to predict potential drug-target interactions. The difficulties of developing computational methods for the prediction of such potential interactions lie in the rarity of known drug-protein interactions and no experimentally verified negative drug-target interaction sample. Furthermore, target proteins need also to be predicted for some new drugs without any known target interaction information. In addition, drug instances may have multiple entities of one entity type. For example, a drug may have one or more targets and one or more pathways of different types, which requires specific classification algorithms, such as multi-label classification to handle such problems.

\section{LITERATURE SURVEY}

Csermely et al [19] wrote a comprehensive assessment of the analytical tools of network topology and dynamics. They summarized the state-of-the-art use of chemical similarity, protein structure, protein-protein interaction, signaling, genetic interaction and metabolic networks in the discovery of drug targets. Takigawa et al [6] developed a fast, scalable algorithm for mining significantly co-occurring subgraphsubsequence pairs from drug-target interactions. Their method allowed the grouping of drug-target interactions into clusters, covering approximately $75 \%$ of interactions containing approved drugs. These clusters were highly exclusive to each other, being statistically significant and logically implying that each cluster corresponds to a distinguished type of polypharmacology. Kim, Jin, and Lee [20] focused on drugdrug interactions (DDI), their adverse effects (DDIAE) and pharmacological information (DDIPharm), and investigated 
the relationship among chemical structures, side effects, and DDIs from several data sources. They showed that DDI is a promising feature in predicting drug-target interactions. Iwata et al [21] performed a systematic analysis of the correlation between drug side effects and protein domains, which they call "pharmacogenomic features," based on the drug-target interaction network. Chen and Zhang [22] developed a semisupervised learning method NetCBP to predict drug-target interaction using labeled and unlabeled interaction information. Assuming coherent interactions between the drugs ranked by their relevance to a query drug, and the target proteins ranked by their relevance to the hidden target proteins of the query drug, they formulate a learning framework maximizing the rank coherence with respect to the known drug-target interactions.

Gao et al [23] proposed a novel prediction method based on drug/compound ontology information extracted from ChEBI to identify drugs target groups from which the kind of functions of a drug may be deduced. Gönen [24] proposed a novel Bayesian formulation that combines dimensionality reduction, matrix factorization and binary classification for predicting drug-target interaction networks using only chemical similarity between drug compounds and genomic similarity between target proteins. Yamanishi et al [25] investigated the relationship between the chemical space, the pharmacological space and the topology of drug-target interaction networks, and show that drug-target interactions are more correlated with pharmacological effect similarity than with chemical structure similarity. In addition, they developed a new method to predict unknown drug-target interactions from chemical, genomic and pharmacological data on a large scale.

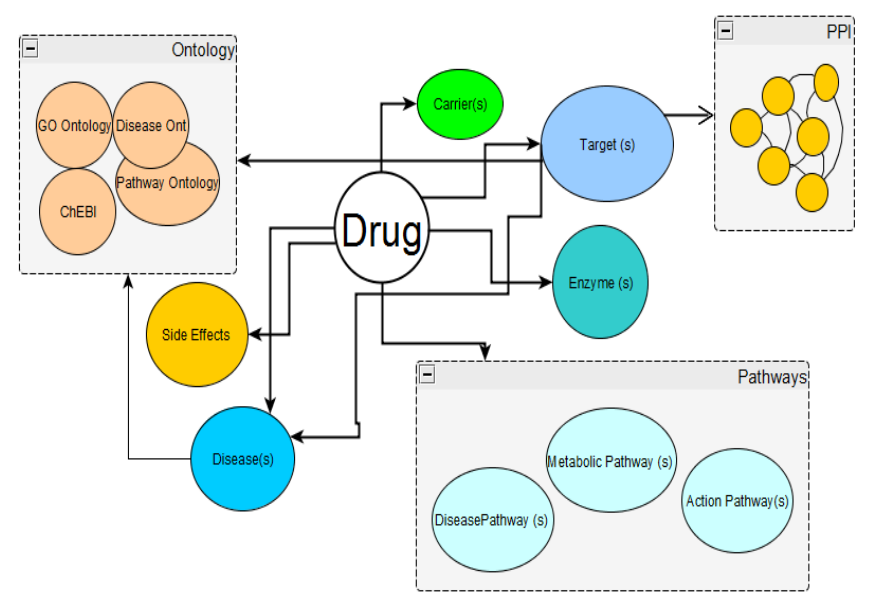

Figure 1: Drug-entities Network

Cheng et al [26] developed three supervised inference methods to predict Drug-Target Interactions and used for drug repositioning, namely drug-based similarity inference (DBSI), target-based similarity inference (TBSI) and network-based inference (NBI). In addition, $\mathrm{Yu}$ et al [27] reported a systematic approach that efficiently integrates the chemical, genomic, and pharmacological information for multi drug targeting and discovery on a large scale, based on two powerful methods of Random Forest (RF) and Support Vector
Machine (SVM). Finally, Singh-Blom et al [28] presented two methods for predicting gene-disease associations based on functional gene associations and gene-phenotype associations in model organisms, which is close to this work. Those methods are: the Katz measure, used in social network link prediction, and CATAPULT (Combining dATa Across species using Positive-Unlabeled Learning Techniques, a supervised machine learning method that uses a biased support vector machine, where the features are derived from walks in a heterogeneous gene-trait network.

\section{METHODOLOGY}

\section{A. Datasets}

The dataset used in this work is collected from various databases, such as Drugbank.ca [1], KEGG DISEASE, KEGG DRUG, KEGG PATHWAY, KEGG BRITE [2], Uniprot [29], and SMPDB [3,4]. The DrugBank.ca database is a unique bioinformatics and cheminformatics resource that combines detailed drug (i.e. chemical, pharmacological and pharmaceutical) data with comprehensive drug target (i.e. sequence, structure, and pathway) information. The database contains 7740 drug entries including 1584 FDA-approved small molecule drugs, 157 FDA-approved biotech (protein/peptide) drugs, 89 nutraceuticals and over 6000 experimental drugs. Additionally, 4282 non-redundant protein (i.e. drug target/enzyme/transporter/carrier) sequences are linked to these drug entries. Each DrugCard entry contains more than 200 data fields with half of the information being devoted to drug/chemical data and the other half devoted to drug target or protein data. In this study, a subset of approved small molecules drugs is used.

SMPDB (The Small Molecule Pathway Database) is an interactive, visual database containing more than 600 small molecule pathways found in humans. SMPDB is designed specifically to support pathway elucidation and pathway discovery in metabolomics, transcriptomics, proteomics and systems biology. All SMPDB pathways include information on the relevant organs, subcellular compartments, protein cofactors, protein locations, metabolite locations, chemical structures and protein quaternary structures. Each small molecule is hyperlinked to detailed descriptions contained in the HMDB or DrugBank and each protein or enzyme complex is hyperlinked to UniProt.

In this work, 440 drugs are used with 12 interaction types. Those interaction types are bleeding risk, urokinase, anemia risk, theophylline, serious infections, immunosuppressive, gastrointestinal ulceration, bleomycin effect, neuropathy, alteplase, serum concentration, and secretin effect. Number of pathways in this study is 60 (161 instances), having three pathway types: amino acids, carbohydrates, and metabolic other acids as classified by Kegg Brite. Each drug may have one or more pathways of action pathways, disease pathways, and metabolic pathways. Sometimes it does not have any data. In the current paper, only metabolic pathways are considered. For example, for the drug Biotin has 14 metabolic pathways, 9 targets, and 45 disease pathways, as shown in Figure 2. 


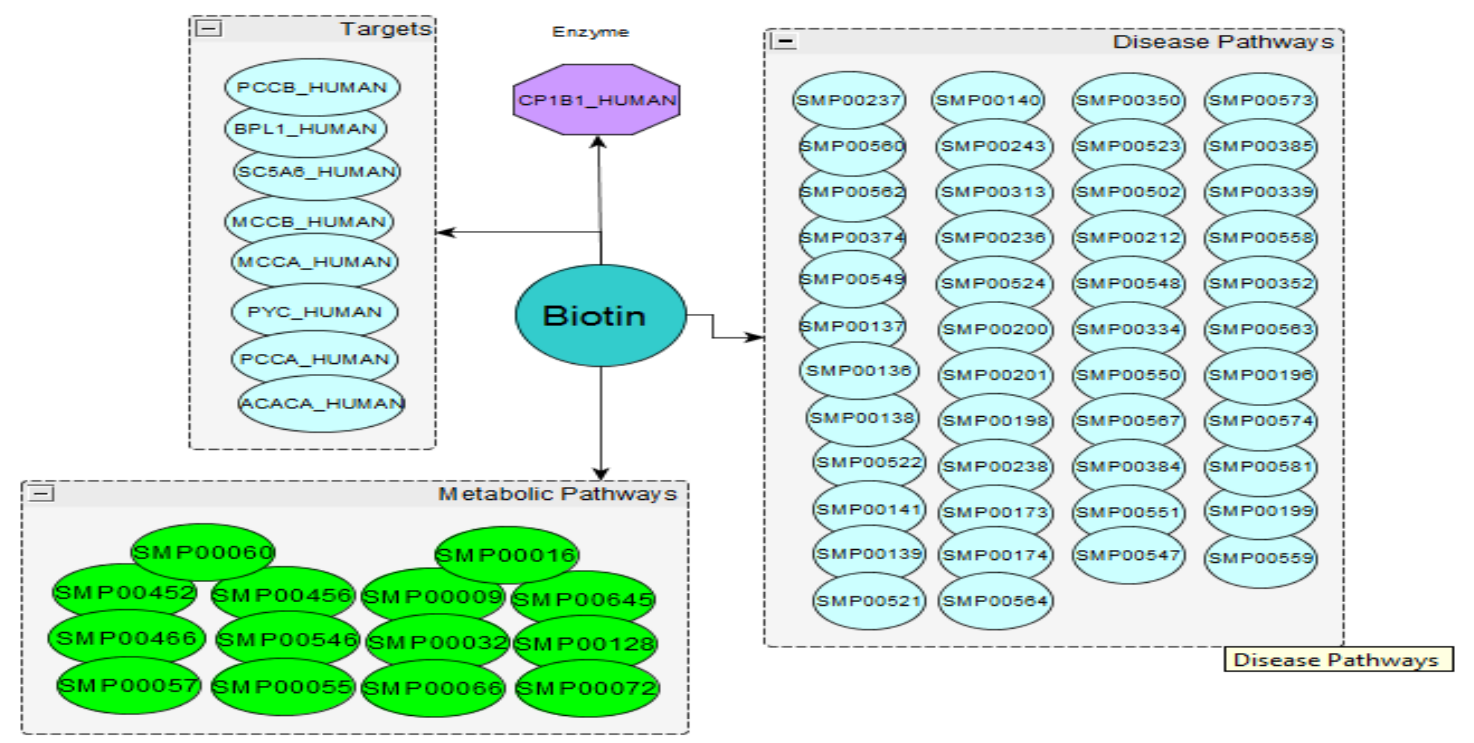

Figure 2: Example of Multi-Labels for Biotin Drug (Drugbank: DB00121) of Multi-Targets, Multi-Disease Pathways, and MultiMetabolic Pathways

However, Biotin drug does not interact with any other drugs or have side effects. The main objective of this work is to classify multi drug-pathway relations. The disease-gene relations will be discussed in future work.

\section{B. Heterogeneous Networks}

Multi-dimensional mining with a focus on homogenous data, such as directed graph alignment [30], undirected graph alignment [31], relational data mining [32], social networks mining [33] are several examples. Recently, such approaches found applications in computational biology [34-36]. However, they have their own limits due to the high complexity of biological networks. In addition, biological networks, social networks, information networks, systems biolgogy, and communication networks include heterogeneous information that give more insights into networks. The extraction of subnetworks (or patterns) involves several relations between the objects of interest, where each such relation is given as a network. The complexity of a particular mining problem increases with the varied nature of the networks, their number, their size, the topology of the requested pattern, and the criteria to optimize [37]. Fertin, Mohamed-Babou, and Rusu [38] presented two networks, respectively, as a directed acyclic graph and an undirected graph, on the same vertex set. In addition, they proposed heuristic and a branch-and-bound algorithm for subnetwork mining.

\section{Multi-label Classification}

Multi-label classification is a challenging problem in many real-world application domains, where each instance can be assigned simultaneously into multiple classes. A typical application of multi-label classification is bioinformatics and drug discovery informatics. For instance, a protein can have one or more functions and a drug can interact with one or more protein targets and have more than one pathway, as illustrated in Figure 2. A pathway can be a drug action pathway, a disease pathway, and a metabolic pathway. In each case, a drug can have one or more drug action pathway, one or more disease pathway, and one or more metabolic pathways. Many methods for multi-label classification have been proposed in the literature.

There are mainly two methods for multi-label classification problems: (1) problem transformation method and (2) algorithm adaptation method. Problem transformation method transfers multi-label problems into single label problems. Algorithm adaptation method extends specific learning algorithm to handle multi-label problems or develop new algorithms to solve this problem. In [39], three algorithms based on the concept of Predicting Clustering Trees (PCT) to deal with HMC problems are compared, namely Clus-HMC [40], Clus-SC and Clus-HSC. As in [41], the authors employ the Euclidean distance as the proximity measure for creating PCTs. The work of Aleksovski et al [42] investigates the use of other proximity measures, such as Jaccard, SimGIC and ImageClef. Ensemble of PCT-based classifiers is also investigated in [43-45]. Sangsuriyun et al [46] propose a global method for HMC, namely Hierarchical Multi-Label Associative Classification (HMAC). Guo and Gu proposed in [47] a multi-label classifier based on conditional dependency networks, where their work showed very good results; hence, their work is somewhat relevant to this paper. However, Zhou and Liu [48] presented an activity-edge centric multi-label classification framework for analyzing heterogeneous information networks with three unique features.

\section{Probabilistic Heterogeneous Network Multi-label Classifier}

The problem of mining drug-related data relies in the data representation and how to perform the prediction tasks accurately. The overall procedure is shown in Figure 3. In the current work, the data is represented as a directed graph, 
denoted $\mathrm{G} . \mathrm{G}=\{\mathrm{V}, \mathrm{E}\}$, where $\mathrm{V}$ represents the common vertex set and $\mathrm{E}$ represents the set of directed edges between vertices with a distribution $\mathrm{P}=\left\{\mathrm{p}\left(\mathrm{fi} \mid \mathrm{pa}_{\mathrm{i}}\right), \forall_{\mathrm{i}}\right\}$ [47] over a set of random variables $\mathrm{F}=\left\{\mathrm{F}_{1}, \mathrm{~F}_{2}, \ldots, \mathrm{F}_{\mathrm{m}}\right\}$ and $\mathrm{m}$ is the number of variables. Each variable $f_{i}$ corresponds to one node $v_{i} V$. The parents of $f_{i}$, denoted as Pai, are the set of nodes vj such that $\left(\mathrm{v}_{\mathrm{j}}, \mathrm{v}_{\mathrm{i}}\right) \in \mathrm{E}$. The array of class of events is denoted as an array of Boolean variables $\mathrm{C}=\left(\mathrm{C}_{1}, \ldots, \mathrm{C}_{\mathrm{n}}\right)$, which expresses whether each class label is relevant or not for the given instance. Thus, $C$ takes its values in $\{0,1\} \mathrm{n}$. An instantiation of class events is denoted as $\mathrm{c}=\left\{\mathrm{c}_{1}, \ldots, \mathrm{c}_{\mathrm{n}}\right\}$; an instantiation of the features is denoted as $\mathrm{f}=\left(\mathrm{f}_{1}, \ldots, \mathrm{f}_{\mathrm{m}}\right)$. A set of complete training instances $S=\{(\mathrm{c} \mid \mathrm{f})\}$ is supposed to be available. In this case, F represents the set of drugs D and their features and $\mathrm{C}$ are pathways. The joint probability distribution (JPD) of $\mathrm{F}$ can then be written as (as in Bayesian probability):

$$
p\left(f_{1}, f_{2}, \ldots, f_{m}\right)=\prod_{i=1}^{m} p_{i}\left(f_{i} \mid C i\right)
$$

Features considered for each drug are the chemical structure drug similarity, drug-drug interactions, and proteinprotein similarity; the whole procedure is illustrated in Figure 3 and the algorithm is shown in Table 1.

Chemical Structure Drug Similarity (CS): chemical structures of drugs are obtained from drugbank, where the similarity is computed. DrugBank has several formats for representing chemical structures, such as canonical smiles, mole files, and InChI keys. In this paper, the canonical smiles format is used to represent the chemical structure, where the SIMCOMP KEGG tool is applied for comparison [2].

Drug-Drug Interaction: The drug-drug interaction is computed if there is an interaction between two drugs. In G, if there is an interaction between two drugs there is edge drawn between drug $\mathrm{i}$ and drug $\mathrm{j}$. Figure 4 illustrates a subset of the interaction between drugs, taken from DrugBank [1] database. The computation of such an interaction is expressed as follows:

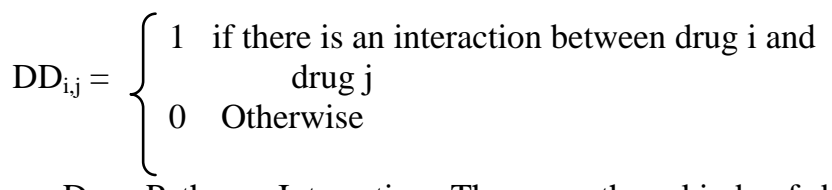

Drug-Pathway Interaction: There are three kinds of drugpathway interaction, as mentioned previously, the drug-action pathway, drug metabolite pathway, and drug disease pathway. The computation of interactions is as follows:

$\mathrm{DP}_{\mathrm{g}, \mathrm{t}}=\left\{\begin{array}{cc}1 & \begin{array}{c}\text { if there is a relation between drug } \mathrm{g} \text { and } \\ \text { pathway } \mathrm{t}\end{array} \\ 0 & \text { Otherwise }\end{array}\right.$

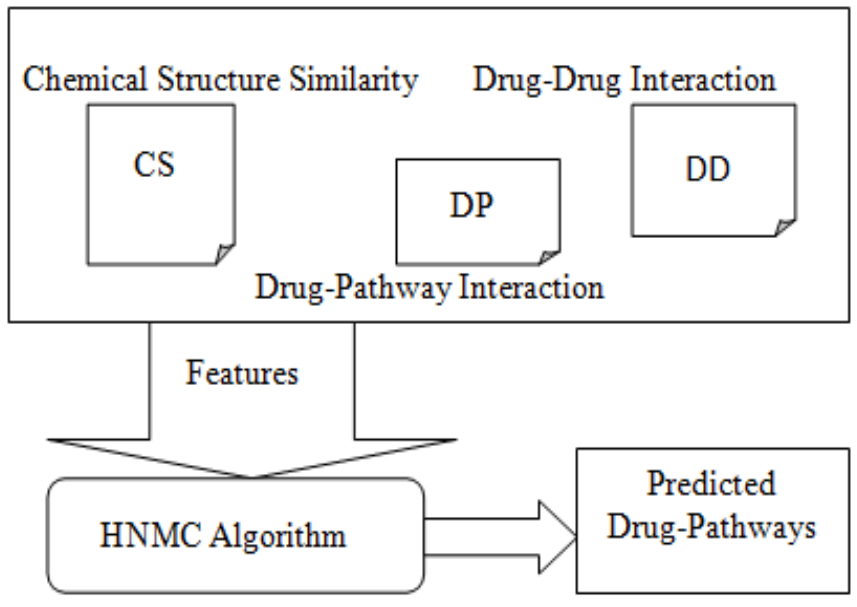

Figure 3: Prediction Process of Multi Drug-Pathways

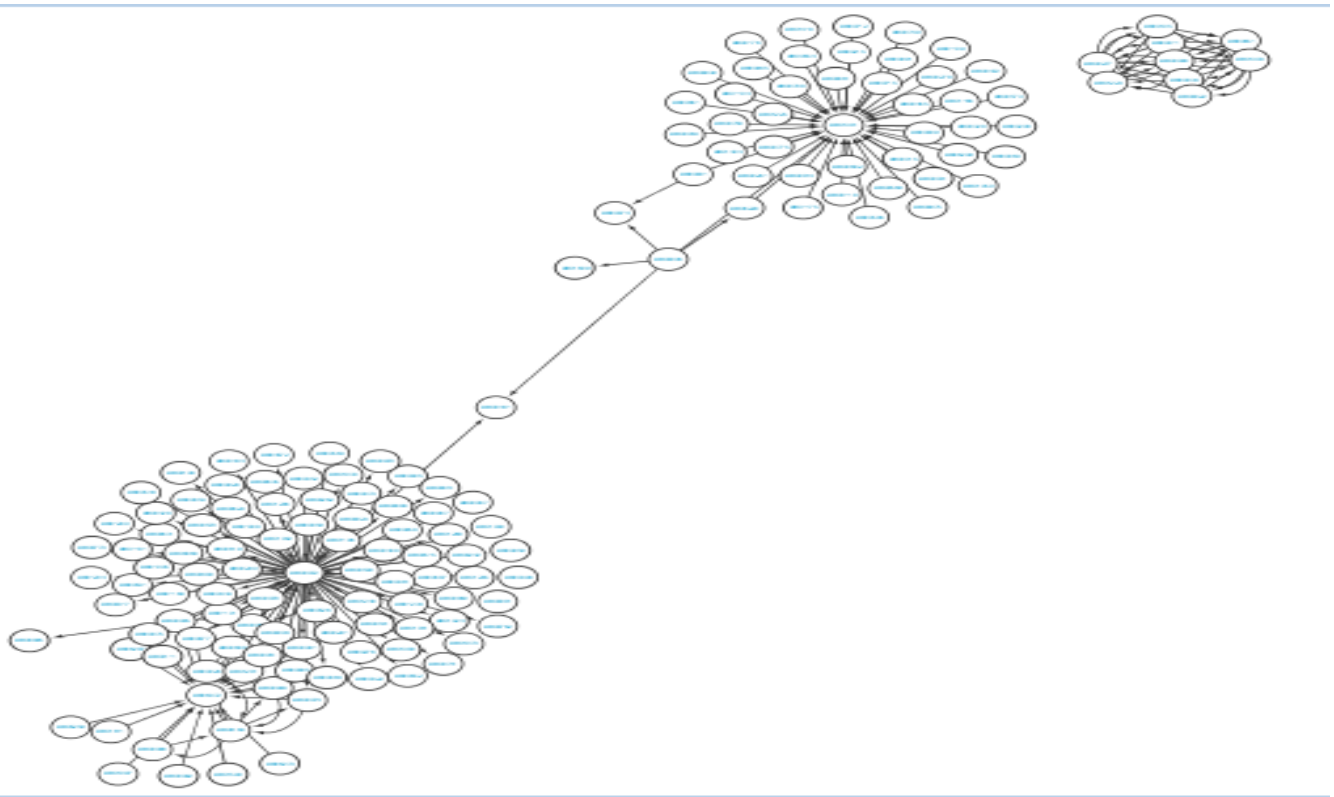

Figure 4: Subset of Drug-Drug Interaction Network (using CytoScape) 
Table 1: HNMC Algorithm

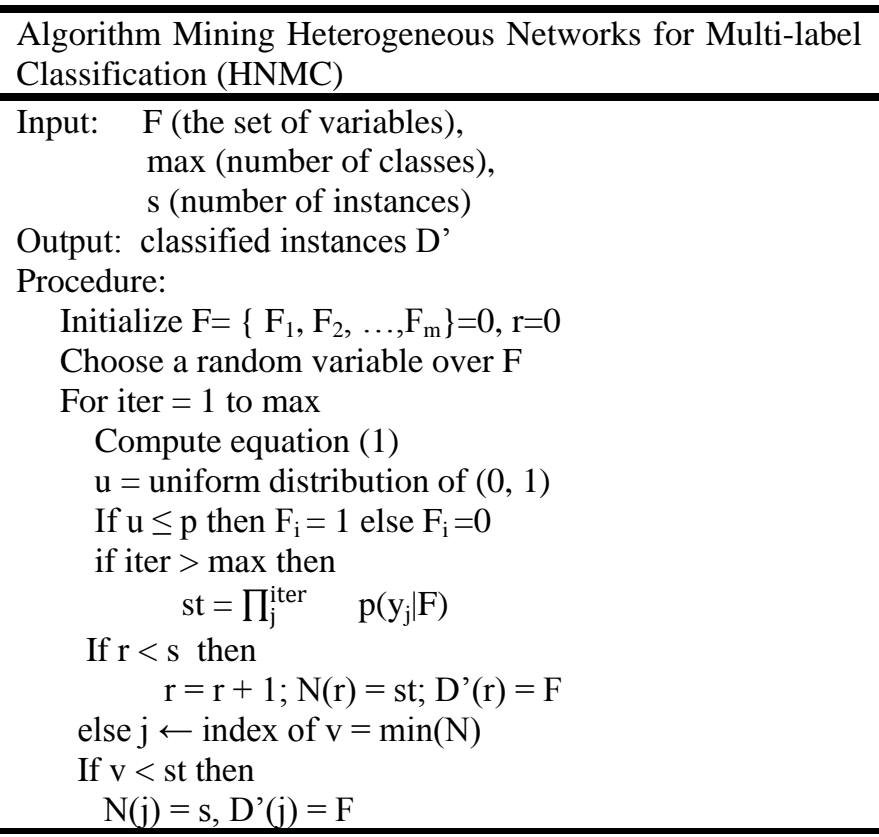

\section{EXPERIMENTAL RESULTS}

Prediction of multi drug-pathways is performed using the HNMC algorithm. The dataset is described previously in section 3. Multiple-label classification is based on chemical structure similarity, based on drug-drug interaction including their interaction type (DDI), and based on drug-pathway interactions (DPI) is accomplished. Table 2 shows parts of the datasets taken for drug-drug interactions; Table 3 shows parts of drug-pathways taken in this study.

The results of prediction accuracy, precision, recall, F1 measure, where precision is computed as in equation (2), Recall as in equation (3), F1-measure as in equation (4), respectively, as follows:

$$
\text { Precision }=\frac{\mathrm{TP}}{\mathrm{TP}+\mathrm{FP}}
$$

$$
\begin{gathered}
\text { Recall }=\frac{\mathrm{TP}}{\mathrm{TP}+\mathrm{FN}} \\
\mathrm{F} 1=\frac{2 \mathrm{TP}}{2 \mathrm{TP}+\mathrm{FP}+\mathrm{FN}}
\end{gathered}
$$

From Table 4, one can see results of the proposed algorithm. An average of $90 \%$ precision, $92.35 \%$ recall, $92 \%$ accuracy, and $96 \%$ ROC were obtained.

Table 2: Subset of Drug-Drug Interaction Datasets

\begin{tabular}{|l|l|l|}
\hline $\begin{array}{l}\text { Drug1 } \\
\text { (drugbank_ID) }\end{array}$ & $\begin{array}{l}\text { Drug2 } \\
\text { (drugbank_ID) }\end{array}$ & Interaction_Type \\
\hline $\begin{array}{l}\text { DB01418 } \\
\text { (Acenocoumarol) }\end{array}$ & $\begin{array}{l}\text { DB01381 (Ginkgo } \\
\text { Biloba) }\end{array}$ & Bleeding \\
\hline $\begin{array}{l}\text { DB06209 } \\
\text { (Prasugrel) }\end{array}$ & $\begin{array}{l}\text { DB01381 (Ginkgo } \\
\text { Biloba) }\end{array}$ & Bleeding \\
\hline $\begin{array}{l}\text { DB06228 } \\
\text { (Rivaroxaban) }\end{array}$ & $\begin{array}{l}\text { DB01381 (Ginkgo } \\
\text { Biloba) }\end{array}$ & Bleeding \\
\hline $\begin{array}{l}\text { DB00001 } \\
\text { (Lepirudin) }\end{array}$ & $\begin{array}{l}\text { DB00374 } \\
\text { (Treprostinil) }\end{array}$ & Anemia \\
\hline $\begin{array}{l}\text { DB00004 } \\
\text { (Denileukin } \\
\text { diftitox) }\end{array}$ & $\begin{array}{l}\text { DB06372 } \\
\text { (Rilonacept) }\end{array}$ & Anemia \\
\hline
\end{tabular}

\section{CONCLUSION AND Future ENHANCEMENTS}

Drugs side effects affect our health tremendously. Drugrelated information is very important to mine in order to discover hidden relations such as multi drug -pathways, multidrug targets, and drug-disease relations. In this work, a probabilistic heterogeneous network multi-label classifier was proposed to classify multi drug-pathways. Two datasets were considered: one is based on Drug-Drug Interaction (DDI) and the other was based on Drug-Pathways Interactions (DPI). With an average of $90 \%$ precision, $92.35 \%$ recall, $92 \%$ accuracy, and 96\% ROC were achieved. Future work includes enlarging the datasets for drug-pathway prediction and applying this algorithm on Mapreduce since the data is very huge and big data requires reducing the functions to be performed. In addition, mining the other parts of drug network to discover hidden knowledge in drug-disease and multi drug-targets will be considered.

Table 3: Subset of Drug-Pathway Datasets

\begin{tabular}{|l|l|l|l|l|l|l|}
\hline SMPDB & Metabolite & KEGG & ChEBI & DrugBank & PathwayType & Class \\
\hline SMP00055 & HMDB00030 & C00120 & 15956 & DB00121 & Metabolic & Amino_acid \\
\hline SMP00055 & HMDB00119 & C00048 & 16891 & DB04343 & Metabolic & Amino_acid \\
\hline SMP00055 & HMDB00123 & C00037 & 15428 & DB00145 & Metabolic & Amino_acid \\
\hline SMP00067 & HMDB00056 & C00099 & 16958 & DB03107 & Metabolic & Amino_acid \\
\hline SMP00067 & HMDB00134 & C00122 & 18012 & DB01677 & Metabolic & Amino_acid \\
\hline SMP00057 & HMDB00072 & C00417 & 32805 & None & Metabolic & Carbohydrate \\
\hline SMP00057 & HMDB00030 & C00120 & 15956 & DB00121 & Metabolic & Carbohydrate \\
\hline
\end{tabular}


Table 4: Results of HNMC Algorithm

\begin{tabular}{|c|c|c|}
\hline Measures & $\begin{array}{c}\text { DDI } \\
440 \text { instances } \\
12 \text { distinct classes }\end{array}$ & $\begin{array}{c}\text { DPI } \\
161 \text { instances } \\
3 \text { distinct classes }\end{array}$ \\
\hline Precision & $87 \%$ & $93 \%$ \\
\hline Recall & $92 \%$ & $93 \%$ \\
\hline F1 & $89 \%$ & $93 \%$ \\
\hline Accuracy & $92 \%$ & $92.7 \%$ \\
\hline ROC & $94 \%$ & $98 \%$ \\
\hline
\end{tabular}

Table 5: AUC of HNMC Compared with Other Algorithms

\begin{tabular}{|l|l|l|l|l|}
\hline Algorithm & AUC & $\begin{array}{l}\text { Single } \\
\text { Drug-target }\end{array}$ & $\begin{array}{l}\text { Multiple } \\
\text { Drug-target }\end{array}$ & Multiple Drug-Pathway \\
\hline HNMC (DDI) & $94 \%$ & No & No & Yes \\
\hline HNMC (DPI) & $98 \%$ & No & No & Yes \\
\hline $\begin{array}{l}\text { Kim, Jen, and Lee [20] } \\
\text { (KL1LR Algorithm STITCH DDI) }\end{array}$ & $75 \%$ & Yes & No & No \\
\hline $\begin{array}{l}\text { Kim, Jen, and Lee [20] } \\
\text { (KL1LR Algorithm } \\
\text { KEGG DDI) }\end{array}$ & $73 \%$ & Yes & No & No \\
\hline $\begin{array}{l}\text { Kim, Jen, and Lee [20] } \\
\text { (SVM Algorithm } \\
\text { STITCH DDI ) }\end{array}$ & $79 \%$ & Yes & No & No \\
\hline $\begin{array}{l}\text { Kim, Jen, and Lee [20] } \\
\text { (SVM Algorithm } \\
\text { KEGG DDI) }\end{array}$ & $69 \%$ & Yes & No & No \\
\hline $\begin{array}{l}\text { Chen \& Zhang [22] } \\
\text { (Enzymes) }\end{array}$ & $82 \%$ & Yes & No & No \\
\hline $\begin{array}{l}\text { Chen \& Zhang [22] } \\
\text { (ion channels) }\end{array}$ & $80 \%$ & Yes & No & No \\
\hline $\begin{array}{l}\text { Chen \& Zhang [22] } \\
\text { (GPCRs) }\end{array}$ & $82 \%$ & Yes & No & No \\
\hline $\begin{array}{l}\text { Chen \& Zhang [22] } \\
\text { (nuclear receptors) }\end{array}$ & $83 \%$ & Yes & No & No \\
\hline Yu et al [27] & No & Yes & No \\
\hline
\end{tabular}

\section{REFERENCES}

[1] www.drugbank.ca/ (last accessed: October 31, 2014)

[2] www.genome.jp/kegg/ (last accessed: October 31, 2014)

[3] D.S. Wishart, A. Frolkis, C. Knox, et al., "SMPDB: The Small Molecule Pathway Database", Nucleic Acids Res.Jan; Vol. 38(Database issue):D480-7, 2010.

[4] T. Jewison, Y. Su, FM. Disfany, et al., "SMPDB 2.0: Big Improvements to the Small Molecule Pathway Database", Nucleic Acids Res., Vol. 42, 2013.

[5] J. Tang and T. Aittokallio, "Network Pharmacology Strategies Toward Multi-Target Anticancer Therapies: From Computational Models to Experimental Design Principles," Current Pharmaceutial Design, Vol. 20, Pp.23-26, 2014.

[6] I. Takigawa, K. Tsuda, H. Mamitsuka, "Mining Significant Substructure Pairs for Interpreting Polypharmacology in Drug-Target Network," PLOS One, February 23, Vol. 6, Issue No. 2, Pp.1-10, 2011.

[7] A. Hopkins, "Network Pharmacology: the Next Paradigm in Drug Discovery", Nat Chem Biol, Vol. 4: Pp.682-690, 2008.

[8] B. Apsel, J. Blair, B. Gonzalez, T. Nazif, M. Feldman, et al., "Targeted Polypharmacology: Discovery of Dual Inhibitors of Tyrosine and Phosphoinositide Kinases", Nature Chemical Biology, Vol. 4: Pp.691699, 2008.

[9] M. Campillos, M. Kuhn, A. Gavin, L. Jensen, P. Bork, "Drug Target Identification Using Side-effect Similarity", Science, Vol. 321: Pp.263266, 2008.

[10] S. Frantz, "Drug Discovery: Playing Dirty", Nature, Vol. 437, Pp. 942 943, 2005.

[11] M. Yildirim, K. Goh, M. Cusick, A. Baraba'si, M. Vidal, "Drug-target Network", Nat Biotechnology, Vol.25: Pp.1119-1126, 2007.

[12] https://pubchem.ncbi.nlm.nih.gov/

[13] http://www.ncbi.nlm.nih.gov/pubmed
[14] http://www.drugbank.com

[15] http://sideeffects.embl.de/

[16] http://www.ebi.ac.uk/chebi/

[17] https://www.pharmgkb.org/

[18] A. Masoudi-Nejad, Z. Mousavian, and J. Bozorgmehr, "Drug-target and Disease Networks: Polypharmacology in the Post-genomic Era", In Silico Pharmacology, 1:17, 2013.

[19] P. Csermely, T. Korcsmáros, H. Kiss, G. London, R. and Nussinov, "Structure and Dynamics of Molecular Networks: A Novel Paradigm of Drug Discovery: A Comprehensive Review", Pharmacology \& Therapeutics, Vol. 138, Pp.333-408, 2013.

[20] S. Kim, D. Jin, and H. Lee, "Predicting Drug-Target Interactions Using Drug-Drug Interactions", PLOS One, Vol.8, Issue 11, 2013.

[21] H. Iwata, S. Mizutani, Y. Tabei, M. Kotera, S. Goto, and Y. Yamanishi, "Inferring Protein Domains Associated with Drug Side Effects Based on Drug-target Interaction Network", BMC Systems Biology, Vol. 7, 2013.

[22] H. Chen and Z. Zhang, "A Semi-Supervised Method for Drug-Target Interaction Prediction with Consistency in Networks," PLoS ONE, Vol. 8(5), May 2013

[23] Y. Gao, "Prediction of Drugs Target Groups Based on ChEBI Ontology", BioMed Research International, Volume 2013.

[24] M. Gönen, "Predicting drug-target interactions from chemical and genomic kernels using Bayesian matrix factorization”, Bioinformatics, Vol. 28, no. 18, Pp.2304-2310, 2012.

[25] Y. Yamanishi, M. Kotera, M. Kanehisa, and S. Goto, "Drug-target Interaction Prediction from Chemical, Genomic and Pharmacological Data in an Integrated Framework", Bioinformatics, Vol. 26, i246-i254, 2010

[26] F. Cheng, "Prediction of Drug-Target Interactions and Drug Repositioning via Network-Based Inference", PLOS Computational Biology, Vol. 8(5) (2012). 
[27] H. Yu, J. Chen, X. Xu, Y. Li, H. Zhao, Y. Fang, X. Li, W. Zhou, W. Wang, Y. Wang, "A Systematic Prediction of Multiple Drug-Target Interactions from Chemical, Genomic, and Pharmacological Data", PLOS One, Vol. 7, Issue 5, 2012.

[28] U. Singh-Blom, N. Natarajan, A. Tewari, J. Woods, I. Dhillon, and E. Marcotte, "Prediction and Validation of Gene-Disease Associations Using Methods Inspired by Social Network Analyses", PLOS One, Vol. 8(5), 2013.

[29] http://www.uniprot.org/

[30] H. Bunke, "Graph Matching: Theoretical Foundations, Algorithms and Applications", In Proc. Vision Interface, 2000:Pp.82-88, 2000.

[31] D. Conte, P. Foggia, C. Sansone, and M. Vento, "Thirty Years of Graph Matching in Pattern Recognition", International Journal of Pattern Recognition and Artificial Intelligence, Vol. 18:Pp.265-298, 2004.

[32] S. Dzeroski and N. Lavrac, Relational data mining. Springer, 2001.

[33] Y. Matsuo, M. Hamasaki, H. Takeda, J. Mori, D. Bollegara, Y. Nakamura, T. Nishimura , K. Hasida, and M. Ishizuka, "Spinning Multiple Social Networks for Semantic Web", In Proc. of the TwentyFirst National Conference on Artificial Intelligence, 2006.

[34] B. Kelley, B. Yuan, F. Lewitter, R. Sharan, B. Stockwell, and T. Ideker, "Pathblast: a Tool for Alignment of Protein Interaction Networks", Nucleic Acids Research, Vol. 32:Pp.83-88, 2004.

[35] R. Sharan, S. Suthram, R. Kelley, T. Kuhn, S. Mccuine, P. Uetz, T. Sittler, R. Karp, and T. Ideker, "Conserved Patterns of Protein Interaction in Multiple Species", National Academy of Sciences, Vol. 102(6):Pp.1974-1979, 2005.

[36] S. Wernicke, and F. Rasche, "Simple and Fast Alignment of Metabolic Pathways by Exploiting Local Diversity", Bioinformatics, Vol. 23:Pp.1978-1985, 2007.

[37] G. Fertin, H. Mohamed-Babou, and I. Rusu, "Algorithms for Subnetwork Mining in Heterogeneous Networks", 11th Symposium on Experimental Algorithms (SEA 2012), Bordeaux, France. Springer, 7228, Pp.184-194, Lecture Notes in Computer Science, 2012.

[38] N. Al Aydie, "Hierarchical Multi-label Classification for Protein Function Prediction Going Beyond Traditional Approaches", Ph.D. Thesis, Department of Computer Science, Wayne University, 2012.

[39] C. Vens, J. Struyf, L. Schietgat, S. Dzeroski, and H. Blockeel, "Decision Trees for Hierarchical Multi-Label Classiffication", Machine Learning, Vol. 73, Issue 2, Pp.185-214, 2008.

[40] H. Blockeel, M. Bruynooghe, S. Dzeroski, J. Ramon, and J. Struyf, "Hierarchical Multi-classification", In Workshop on Multi-Relational Data Mining, Pp. 21-35, 2002.

[41] D. Aleksovski, D. Kocev, and S. Dzeroski, "Evaluation of Distance Measures for Hierarchical Multilabel Classification in Functional Genomics", In Workshop on Learning from Multi-Label Data, Pp. 5-16, 2009.

[42] L. Schietgat, C. Vens, J. Struyf, H. Blockeel, D. Kocev, and S. Dzeroski, "Predicting Gene Function Using Hierarchical Multi-Label Decision Tree Ensembles", BMC Bioinformatics, Vol. 11, Issue2, Pp.1-14, 2010.

[43] I. Dimitrovski, D. Kocev, S. Loskovska, and S. Deroski, "Hierarchical Annotation of Medical Images", Pattern Recognition, Pp.2436-2449, 2011.

[44] I. Dimitrovski, D. Kocev, S. Loskovska, and S. Dzeroski, "ImageCLEF Medical Image Annotation Task: PCTs for Hierarchical Multi-Label Classification", In CLEF, Pp.231-238, 2009.

[45] I. Dimitrovski, D. Kocev, S. Loskovska, and S. Dzeroski, "Detection of Visual Concepts and Annotation of Images Using Ensembles of Trees for Hierarchical Multi-Label Classification”, In ICPR, Pp.152-161, 2010.

[46] S. Sangsuriyun, S. Marukatat, and K. Waiyamai, "Hierarchical MultiLabel Associative Classification (HMAC) Using Negative Rules", In International Conference on Cognitive Informatics, Pp. 919-924, 2010.

[47] Y. Guo, and S. Gu, "Multi-Label Classification Using Conditional Dependency Networks", Proceedings of the Twenty-Second International Joint Conference on Artificial Intelligence, Pp. 1300-1305, 2011.

[48] Y. Zhou, and L. Liu, "Activity-edge Centric Multi-label Classification for Mining Heterogeneous Information Networks", Proceedings of the 20th ACM SIGKDD Conference on Knowledge Discovery and Data Mining (KDD'14), 2014.

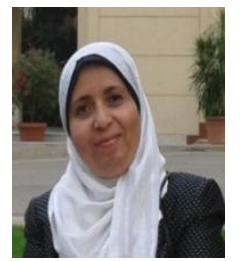

Taysir Hassan A. Soliman was born in Assiut, Egypt in 1970. She obtained her B.Sc. in Computer Science from Marymount University, USA in 1990. She got her Ph.D. in Bioinformatics in 2004 from Faculty of Computers \& Information Science, Ain Shams University, Egypt on a Joint Supervision with University of Connecticut, USA. She is currently working as an Associate Professor and Head of Information Systems Department, Faculty of Computers \& Information, Assiut University, Egypt. Research interests are data mining and bioinformatics. A visiting scientist at IBM, CAS Center, Cairo Division, May 2005-2007. A member at IEEE and ACM SIGKDD, and ACM SIGBIO. A supervisor of Bioinformatics Research group at the Faculty. Last two publications are: 1) Taysir Hassan Soliman, M. A. Elmasry, A. Hedar, M. M. Doss, "Sentiment Analysis of Arabic Slang Comments on Facebook," International Journal of Computers \& Technology, Vol. 12, No. 5, 2014. 2) Ibrahim E El-Semman, Fredrik H Karlsson, Saeed Shoaie, Intawat Nookaew,Taysir H Soliman, and Jens Nielsen, "Genome-scale metabolic reconstructions of Bifidobacterium adolescentis L2-32 and Faecalibacterium prausnitzii A2-165 and their interaction," BMC Systems Biology, 8:41, 2014 (highly accessed). 